\title{
COATING OF FOODS WITH PLANT BASED GUM TO REDUCE OIL ABSORPTION DURING FRYING
}

\author{
By \\ Oushadhee Pubudika Weerasekera
}

This Thesis submitted in partial fulfillment of the requirements for the Degree of Masters of Food Science and Technology,

Department of Food Science and Technology,

Faculty of Applied Science,

University of Sri Jayewardenepura, Sri Lanka 


\section{DECLARATION}

The work described in this thesis was carried by me, under the supervision of Dr. (Mr.)

S.B Navarathne and the report on this thesis has not been submitted in whole or in part of any University or any other institution for another Degree/ Diploma.

14.07. 2015

Whel

Date

O.P Weerasekera 
"I certify that the above statement made by the candidate is true and that this thesis is suitable for submission to the university for the purpose of evaluation"

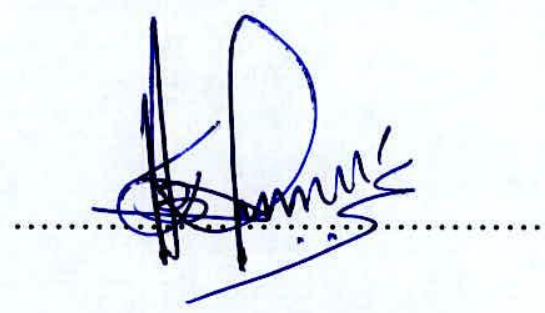

$14 \cdot 07 \cdot 2015$

Dr. (Mr.) S.B Navaratne

Date

Department of Food Science and Technology,

Faculty of Applied Sciences,

University of Sri Jayewardenepura,

Sri Lanka. 
Dedicated to my loving family members 
TABLE OF CONTENT

\section{Content}

Page

LIST OF CONTENT $\quad$ i

LIST OF TABLES $\quad$ iv

LIST OF FIGURES

ACKNOWLEDGEMENT $\quad$ vi

ABSTRACT vii

1. INTRODUCTION 1

1.1 Introduction 1

1.2 Objectives $\quad 3$

1.2.1 General Objectives $\quad 3$

1.2.2 Specific Objectives 3

2. LITERATURE REVIEW 4

2.1 Food Frying $\quad 4$

2.2 Plant Gums 9

2.3 Morphological Features of Selected Plants with Natural Food Gums $\quad 10$

$\begin{array}{ll}\text { 2.3.1 Durian (Duriozibethinus) } & 10\end{array}$

$\begin{array}{ll}\text { 2.3.2 Okra (Abelmoschus esculentus) } & 18\end{array}$

2.3.3 Cinnamon Leaves (Cinnamomum verum) 24

2.3.4 Dawul Kurundu (Neolitsea cassia) 30

3. MATERIALS AND METHODS 34

3.1 Materials 34

3.1.1 Materials used for Extraction of Gums, Coating and Frying 34

3.1.2 Materials used for determination of total oil content 35 
3.1.3 Materials used for analysis of Free Fatty Acid content

3.1.4 Material used for sensory evaluation

3.2 Extraction of Gums and method of Coating

3.2.1 Extraction of Gums

3.2.1.1 Extraction of Durian Seed Gum

3.2.1.1.1 Extraction Process (Solvent) of Durian Seed Gum

3.2.1.1.2 Extraction Process (Water)

3.2.1.2 Extraction of Okra Gum/ Mucilage

3.2.1.2.1 Extraction Process (Water/ Solvent)

3.2.1.3 Extraction of Cinnamon Leaf Gum 39

3.2.1.3.1 Extraction Process (Water/ Solvent) 39

3.2.1.4 Extraction of Davul Kurundu Leaf Gum 39

3.2.1.4.1 Extraction Process (Water/ Solvent) 39

3.2.2 Coating of Gums 40

3.3 Analysis of absorbed total oil Content during frying 41

3.4 Analysis of Free Fatty Acid Content (Acid Value) 41

3.5 Sensory Evaluation 42

4. RESULTS AND DISCUSSION 43

4.1 Extraction Methods and Coating 43

4.1.1 Extraction Methods

4.1.2 Coating of Gums

4.2Rate of Oil absorption and percentage of reduction of oil absorption 46

4.2.1 Amount of oil retained in flasks 46

4.2.2 Percentage of oil absorption 47

4.2.3 Reduction of oil absorption 51

4.3 Determination of Acid Value 
4.3.1 Acid Value of the samples

4.4 Sensory evaluation results

5. CONCLUSION 58

6. REFERENCES

60

7. APPENDICES

66 


\section{LIST OF TABLES}

Table 2.1: Nutrition Facts of Durian Seed 16

Table 2.2: Nutrition Facts of Okra 22

Table 2.3: Nutrition Facts of Cinnamonn Leaf $\quad 30$

Table 2.4: Nutrition Facts of Dawul Kurundu Leaf 32

Table 4.1: Properties of Gums/ Mucilage of selected plants 43

Table 4.2: Weights of the oil retained in the flasks 47

Table 4.3: Percentage of Oil Absorption 48

Table 4.4: Reduction of Oil Absorption relative to the Control 51

Table 4.5: Required $\mathrm{NaOH}$ volume for neutralization 53

Table 4.6: Acid Value of the treatments $\quad 54$

Table 4.7: Summery of Sensory Evaluation results (panelist responses) 55 


\section{LIST OF FIGURES}

Figure 4.1: Interaction Plot of Plant Gums and Type of Oil

Figure 4.2: Reduction of Oil absorption relative to the Control

Figure 4.3: Summery obtained from Fred Mann Test 


\section{ACKNOWLEDGEMENT}

The process of obtaining a MSc degree is not a journey that can be completed alone. I would like to thank the many individuals, named and unnamed, who have helped me throughout this journey. Particularly thanks are owing to my supervisor Dr S.B Navarathne, who guided and advised me through this circuitous process with infinite patience. I would also like to thank Dr. Jagath Wansapala, who is always ready to share with me his vast knowledge.

In addition, I would like to thank almost all the technical staff of the laboratory of Food Science \& Technology for the given support to finish my work successfully.

Then a special thanks goes to my husband Mr Randil Pushpananda, and Ms H.N.D Thilini, $\mathrm{PhD}$ students of University of Colombo, who always supported me to find valuable abstracts during writing up the thesis.

I take this opportunity to thank Mr. Niranga Weerasinghe, who supported me to analyze the results using statistical software.

And last, but not least, I would like to thank my parents Mr \& Mrs Weerasekera. I appreciate your love and support during these years, thank you! This thesis is also the fruit of your hard work. 


\title{
COATING OF FOODS WITH PLANT BASED GUM TO REDUCE OIL ABSORPTION DURING FRYING
}

\author{
by $O$. P. Weerasekera
}

\begin{abstract}
Fried foods are popular due to their taste, distinctive flavour, aroma and crunchy texture. Oil consumption especially saturated fat is considered a major factor increasing health risks, such as coronary heart disease (CHD), cancer, diabetes and hypertension, that risk human life.

One of the most important quality parameters of fried food is the amount of fat absorbed during the process, which undermines recent consumer trends towards healthier food and low-fat products. Several factors affect oil absorption in fried foods, including process conditions ( temperature and residence time), initial moisture content of the product, raw material composition, slice thickness, pre-frying treatment, degree of starch gelatinization prior to frying, and oil quality and coatings.
\end{abstract}

Application of edible gum/ film is one of the important factors, through which reduction of the oil absorption during frying can be ensured. It has been previously reported that the coating with cellular derivatives cause the formation of protective layer on the surface, which eventually decreases the oil uptake in the fried products.

Here, this research discusses four edible plant gums named Durian seed gum, Okra pod gum, Cinnamon leaf gum and DawulKurundu leaf gum. Extracted gums were coated on potato chips by dipping method. Then, they were fried in coconut oil and palm oil separately and measured the absorbed oil content using rapid method of oil extraction. The results were then analyzed using Factorial One Way ANOVA Design. From the statistical analysis, it can be concluded that there is a significant difference between the oil absorption in different coatings as well as in frying medium. Then, the four best samples which had less oil absorption were taken for sensory evaluation. The results were analyzed using Fread Mann Test.

According to the results obtained, Okra pod gum was the most effective gum source, which reduced the oil absorption during frying (34\%). However, from the sensory aspects, it was not the best source for coating of food items. It got lower points from the sensory evaluation in both cases.

Second most effective gum source, which reduced the oil absorption during frying was Durian Seed gum. In coconut oil, as well as in palm oil, it had shown the second most reduction in oil absorption. The results of sensory evaluation proved that the best quality chips are the durian seed gum coated chips.

When the cinnamon leaf gum and dawulkurundu leaf gum are concerned, they both did not show any remarkable reduction in oil absorption during frying. It may be due to releasing of gum to the oil. On the other hand, both gums might be heat unstable. Therefore, the two gums had to be rejected as both are not satisfying the main objective of this research. 


\section{CHAPTER 1}

\section{INTRODUCTION}

\subsection{Introduction}

Frying is cooking of food in oil or another fat, a technique that originated in ancient Egypt around $2500 \mathrm{BC}$. Chemically, oils and fats are the same, differing only in melting point, and the distinction is made only when needed. In commerce, many fats are called oils by custom, e.g. palm oil and coconut oil, which are liquids at room temperature. A variety of foods may be fried, including potato chips, bread, eggs and foods made with eggs, such as omelets or pancakes.

Fats can reach much higher temperatures than water at normal atmospheric pressure. Yet, aside from their high caloric value, fried foods can be nutritious and favorable compared with other cooking methods, such as baking and boiling. Fried foods are popular due to their taste, distinctive flavour, aroma and crunchy texture.

One of the most important quality parameters of fried food is the amount of fat absorbed during the process, which undermines recent consumer trends towards healthier food and low-fat products. Despite its considerable fat content and intensified consumer-awareness of the relationships between food, nutrition and health, frying remains a principal cooking method. Oil consumption, especially saturated fat, is considered a major factor increasing health risks such as coronary heart disease (CHD), cancer, diabetes and hypertension, and even linked to increased causes of deaths. Fried foods contribute to a significant proportion of the total fat consumed in the Western world. 
Moreover, scientists are now concerned about reducing oil content in fried foods in different ways. Knowledge of optimal frying conditions is important to produce French fries with the lowest fat content, as well as under industrial conditions at home.

Several factors affect oil absorption in fried foods, including process conditions temperature and residence time), initial moisture content of the product, raw material composition, slice thickness, pre-frying treatment, degree of starch gelatinization prior to frying, oil quality and coating.

Here, this research discusses one of the important factors, which can reduce the oil absorption during frying. That is the application of edible plant gum over food products prior to frying. Oil uptake in the fried products is determined by two mechanisms i.e. condensation effect and capillary effect, which are altered by the application of different coating materials. The oil uptake by potato chips during frying is largely the function of its surface properties. Therefore, coating is considered as promising route for its mitigation in finished processed product. It has been previously reported that the coating with cellular derivatives cause the formation of protective layer on the surface, which eventually decreases the oil uptake in the fried products. Garcia etal.evaluated the surface application of cellulose derivatives in different formulations for the reduced oil uptake in fried products. He concluded that coating reduce the oil uptake by $35-40 \%$ with appreciable retention of different sensory attributes. Currently, researchers are more interested in developing plant based coating formulations for consumer safety. A variety of plants and plant based materials are being investigated for their usefulness. 


\subsection{Objectives}

\subsubsection{General Objective}

1. Coating of plant based edible gums to reduce oil absorption during frying.

\subsubsection{Specific Objectives}

1. To identify different extraction methods of selected plant gums and types of coating.

2. To determine the rate of oil absorption and percentage of reduction of oil absorption in different plant gums coated potato chips during frying.

3. To determine Acid values of the gum coated fried potato chips.

4. To determine the organoleptic properties of selected gum coated potato chips. 


\section{CHAPTER 2}

\section{LITERATURE REVIEW}

\subsection{Food Frying}

Frying techniques vary in the amount of fat required, the cooking time, the type of cooking vessel required, and the manipulation of the food. Sautéing, stir frying, pan frying, shallow frying, and deep frying are all standard frying techniques.

Sautéing and stir-frying involve cooking foods in a thin layer of fat on a hot surface, such as a frying pan, griddle, wok, or sauté use. Stir frying involves frying quickly at very high temperatures, requiring that the food be stirred continuously to prevent it from adhering to the cooking surface and burning.

Shallow frying is a type of pan frying using only enough fat to immerse approximately one-third to one-half of each piece of food; fat used in this technique is typically only used once. Deep-frying, on the other hand, involves totally immersing the food in hot oil, which is normally topped up and used several times before being disposed. Deepfrying is typically a much more involved process, and may require specialized oils for optimal results.

Deep frying is now the basis of a very large and expanding worldwide industry. Fried products have consumer appeal in all age groups and in virtually all cultures, and the process is quick, can easily be made continuous for mass production, and the food emerges sterile and dry, with a relatively long shelf life. The end products can then be easily packaged for storage and distribution. Examples are potato chips, french fries, nuts, doughnuts, instant noodles, etc. 